\title{
La rationalisation simultanée de l'action et des apprentissages Concepts et exemples
}

\author{
La rationalisation \\ simultanée de l'action et \\ des processus \\ d'apprentissages se \\ caractérise par le fait \\ qu'elle prend explicitement \\ en compte l'objectif \\ d'apprentissage en \\ concevant et en implantant \\ des outils de gestion et des \\ formes d'organisation de \\ I'activité opératoire. Après \\ avoir justifié de l'intérêt et \\ de la nécessité pour les \\ activités complexes, de \\ rationaliser simultanément \\ I'action et les \\ apprentissages, nous \\ mettons en évidence les \\ particularités de deux \\ formes de rationalisation \\ simultanée de l'action et \\ des apprentissages (forme \\ instrumentale et forme \\ organisationnelle), puis \\ nous les illustrons par des \\ études de cas industrielles.
}

$\int \begin{aligned} & \text { ous ne faisons pas preuve d'originalité, en } \\ & \text { disant que depuis plusieurs années, on assiste à } \\ & \text { une complexification croissante du travail et }\end{aligned}$ des activités industrielles en général. Un des faits marquant est l'émergence de ce qu'on dénomme les « technologies intellectuelles » (Pyrard, 1992), qui ne confrontent pas l'opérateur à la matière mais exigent de lui un travail de supervision de processs. Ce changement de la nature du travail se manifeste par l'explosion des aspects purement symboliques de l'intervention humaine. À cette intellectualisation du travail s'ajoute une accélération du changement technologique, ainsi qu'une forte tendance à la «personnalisation » de l'offre (Pine, 1993). Faire face aux impératifs de la variété et des délais devient désormais le lot de nombreuses unités de production. Dans le monde de la conception, l'exacerbation de la concurrence s'est traduite par des exigences de plus en plus fortes en matière de délai, de qualité et de coût, auxquelles s'ajoute aujourd'hui une exigence forte d'innovation.

Aussi, nous partageons le pronostic d'Hatchuel (1995) sur l'avenir de la grande firme industrielle, qui selon lui sera marqué par la rationalisation (c'est-à-dire le 
déploiement de moyens visant l'atteinte d'un objectif donné) des multiples processus d'apprentissage qui construiront à la fois l'organisation et la stratégie de l'entreprise de demain.

Face à ces enjeux, la plupart des acteurs du monde économique considèrent que la compétitivité des entreprises passe avant tout par la maîtrise des dynamiques de connaissances. L'organisation affectera alors une partie de ses ressources humaines à la maîtrise des savoirs, dans le cadre, par exemple, de projets de recherche, ou plus récemment de Knowledge Management et de $«$ e-learning ${ }^{1}$. Ces activités et outils directement et exclusivement dédiés à la création et à la gestion de savoirs, participent clairement d'une rationalisation des processus collectifs d'apprentissage internes à l'entreprise.

En revanche, du fait de l'évolution de leur environnement, de nombreux responsables d'activités industrielles sont amenés à prendre en compte la nécessité de développer de façon endogène de nouveaux savoirs, et ce dans le cadre même des activités opérationnelles de l'entreprise. Il s'agit de situations dans lesquelles les dirigeants ne peuvent se contenter de recruter des employés plus qualifiés ou de faire appel au marché de la formation, mais sont obligés d'assumer la nécessité de réinventer les savoirs de l'action, sans que cela puisse se faire en dehors du cours de l'action. Dans ces situations, il s'agira de concevoir les modes de rationalisation des activités opérationnelles de façon à permettre la genèse de ces savoirs de l'action. Nous avons choisi dans le cadre de cet article de nous intéresser à ces modes de rationalisation. Au sein de l'ensemble des démarches de rationalisation des apprentissages, ces démarches croisant intimement les visées d'action opératoire et d'apprentissage nous semblent mériter une attention particulière. En effet, leurs potentialités sont grandes puisqu'elles visent simultanément (ou du moins cherchent un bon compromis entre) une efficacité à court terme de l'action et une efficacité à long terme grâce à leur propriété de stimulation des apprentissages.

Dans le présent article, nous cherchons: à expliquer brièvement l'intérêt, pour le chercheur en gestion, de l'analyse du lien entre les modes de rationalisation et les processus d'apprentissage organisationnel, à cerner davantage l'idée de la rationalisation simultanée des processus d'action et d'apprentissage: en quoi est-ce une démarche de rationalisation spécifique? à repérer différentes formes de cette rationalisation simultanée. Nous verrons que ces formes sont associées aux leviers d'action qu'on privilégie pour encadrer les apprentissages, qui peuvent être en particulier de nature organisationnelle ou instrumentale. Nous illustrerons ces deux formes de rationalisation des apprentissages par deux études de cas industriels.

\section{I. - ANALYSE DES LIENS ENTRE LES MODES DE RATIONALISATION ET LES PROCESSUS D'APPRENTISSAGE : QUEL INTÉRÊT?}

En tant que chercheur en gestion on en vient naturellement à se poser la question

1. Voir à ce sujet (CERI, 2000), ainsi que (Lefebvre et al., 2001). 
suivante: comment les organisations apprennent à mieux maîtriser leurs activités et donc les savoirs de l'action, sachant que celles-ci doivent s'adapter à un environnement mouvant?

La littérature sociologique, portant en particulier sur l'apprentissage organisationnel ${ }^{2}$, n'apporte pas, malgré son abondance, suffisamment d'éclairage sur cette question. Plus précisément, ces travaux ne permettent pas de bien comprendre les liens entre la nature de l'activité et les dynamiques de développement des connaissances (voir, Sardas et Touati, 1997). À titre d'illustration, certaines grilles de lecture sociologiques des phénomènes d'apprentissage organisationnel adoptent des visions holistes de l'organisation en considérant cette dernière comme un système de traitement de l'information qui interprète l'environnement et enregistre les apprentissages dans la mémoire collective. Bien que séduisantes a priori, ces métaphores n'ont pas de caractère opératoire. D'autres visions théoriques interprètent plutôt les processus d'apprentissage organisationnel comme des processus où les membres de l'organisation agissant à partir de leurs représentations du fonctionnement organisationnel détectent une convergence ou un décalage entre les résultats observés et ceux qui étaient attendus et vont dès lors chercher à corriger l'erreur en inventant de nouvelles stratégies qui vont être codées dans des repères collectifs. Ces travaux encore une fois n'établissent aucun lien entre la forme que peuvent prendre les repères collectifs et la nature de l'activité.

Dans le champ de la gestion, le courant de l'organisation qualifiante ${ }^{3}$ occupe une place importante parmi les travaux qui s'intéressent à la problématique de l'apprentissage organisationnel. Comme son nom l'indique, ce courant de recherche vise à caractériser le caractère apprenant d'une organisation. Selon nous, l'une des lacunes de ce courant de recherche est qu'il ne prête pas assez d'attention à ce qu'on pourrait dénommer l'ingénierie de l'apprentissage. En effet, le concept de l'organisation qualifiante met en avant le fait que le caractère apprenant d'une organisation passe par la communication entre acteurs, l'autonomie du travailleur, l'exploitation des événements (entre autres les incidents) comme occasions d'apprentissage, avec la notion de compétence comme nouveau fondement de la gestion des ressources humaines (Zarifian, 2001). Or, il est évident qu'il ne suffit pas de décréter que les gens doivent communiquer et être autonomes pour qu'une dynamique d'apprentissage se mette en route. Considérant cette question de la communication, il n'est pas du tout sûr qu'une communication non structurée favorise une dynamique de savoirs. Bien entendu, les défenseurs de cette vision prônent le recours à un langage commun pour structurer les échanges. Mais il nous semble qu'il importe de raisonner d'abord en termes de dispositifs de coordination, en s'intéressant à la manière dont s'organise,

2. Sur l'apprentissage organisationnel, voir entre autres: (Hedberg, 1981; Argyris, Schon, 1978; Daft, Weick, 1984 ; Shrivastava, Schneider, 1984; Fiol, Lyles, 1985 ; Levitt, March, 1988; March, 1991; Huber, 1996; Dierkes et al., 2001).

3. Concernant l'organisation qualifiante, voir notamment : Bonnis (1992), Mussano (1992), Veltz et Zarifian (1994), Amadieu, Cadin (1996). 
en fonction des caractéristiques de l'activité, une action collective impliquant des acteurs pouvant très bien parler des langages très différents.

Une autre voie de recherche en gestion autour de la problématique de l'apprentissage s'efforce plutôt d'analyser le rôle des modes de rationalisation dans la dynamique des savoirs ${ }^{4}$. L'expression " modes de rationalisation » recouvre ici tous les dispositifs formels destinés à spécifier les tâches des individus et des groupes (par exemple, une descriptions de poste), à les coordonner (exemple: équipe de gestion de projet), à les évaluer et à les inciter (par exemple, un tableau de bord de suivi des performances).

À titre d'exemple, certains travaux de Moisdon et Weill (1992) dans le domaine de la conception automobile ont montré l'intérêt des plateaux techniques (secteurs dédiés à un produit donné et rassemblant les acteurs provenant de différents métiers) et des procédures de validation (par exemple, le recours à la réalisation de prototypes) comme moyens d'apprentissage collectif. La nature de ces dispositifs de coordination est cohérente avec la nature du travail de conception caractérisé par une recherche de compromis techniques que l'on peut difficilement anticiper. Ces dispositifs favorisent les échanges entre les différents protagonistes du projet et la considération de leurs contraintes mutuelles. L'apprentissage organisationnel devient alors un processus de « prescriptions réciproques » entre acteurs (Hatchuel, 1994).

Une telle articulation de la réflexion sur les processus d'apprentissage avec la réflexion sur les modes de rationalisation permet à notre avis d'aboutir à une interprétation plus riche et plus fine de ces phénomènes. Notre réflexion s'inscrit globalement dans cette perspective où les liens entre nature des savoirs, modes de rationalisation et processus d'apprentissage sont considérés comme centraux. On peut en particulier considérer que chaque mode de rationalisation de l'action est porteur d'une dynamique spécifique d'apprentissage. Mais pour autant, une rationalisation de l'action ne constitue pas nécessairement une rationalisation des apprentissages. Cette dernière est une démarche spécifique que nous allons maintenant caractériser.

\section{II. - LA RATIONALISATION SIMULTANÉE DE L'ACTION ET DES PROCESSUS D'APPRENTISSAGE: EN QUOI EST-CE UNE APPROCHE SPÉCIFIQUE?}

Comme nous l'avons déjà souligné, on pourrait penser a priori que la rationalisation des apprentissages est synonyme de la rationalisation de l'action collective dans la mesure où toute rationalisation de l'action collective est inductrice d'apprentissages individuels croisés. Il suffit par exemple de mettre en place un indicateur de mesure de l'output d'une activité pour que les acteurs concernés acquièrent une nouvelle connaissance utile pour guider leurs actions. Il est devenu d'ailleurs classique d'analyser les modèles de gestion en tant que moyens d'apprentissage (voir, entre autres, Hatchuel et Moisdon, 1993).

4. Voir entre autres : Erschler et Thuriot (1992), Moisdon et Weil (1992), Midler (1993), Ponssard et Tanguy (1993), Aggeri et Hatchuel (1995a); Jeantet et al. (1995); Nakhla, Soler (1996). 
Cependant, nous allons voir que la démarche de rationalisation de l'action revêt une autre logique si elle prend en compte de façon explicite l'objectif d'apprentissage. Nous parlons alors de rationalisation simultanée de l'action et des processus d'apprentissage. À l'inverse des formes traditionnelles de rationalisation de l'action, cette forme particulière de rationalisation est pour nous une démarche dans laquelle les apprentissages sont pensés et organisés d'avance. L'objectif peut être de développer les savoirs de l'action, de les capitaliser et ou de les partager et diffuser au sein de l'organisation. Ceci ne signifie nullement que les formes traditionnelles de rationalisation des activités opératoires n'induisent pas des apprentissages. Seulement, elles n'ont pas comme objectif premier de structurer ces derniers. Ainsi à titre d'illustration, les formes d'organisation matricielles (les équipes projet réunissant des représentants de différentes directions « métier ») dans le domaine de la conception automobile ont été mises en place essentiellement pour accroître la réactivité de l'activité. Cela n'empêche pas qu'elles ont également permis d'avancer dans la résolution des problèmes d'interface, qui ne relèvent pas d'un métier donné (exemple de problème d'interface: l'acoustique). Néanmoins, on ne peut pas prétendre que les savoirs d'interface, dont on voulait améliorer la maîtrise, aient fait l'objet d'une quelconque formalisation ou gestion. Cet exemple montre bien que si toute rationalisation des opérations induit des apprentissages, cet effet d'apprentissage est souvent limité en raison du fait que la démarche de rationalisation en question n'a pas été conçue dans cet esprit.
Cela étant, la rationalisation des apprentissages peut bien entendu rentrer à court terme en conflit avec les visées plus classiques de rationalisation de l'action. C'est la leçon tirée d'une expérience de mise en place d'une assurance qualité pour une activité de conception de réseaux de télécommunication pour des entreprises (David et al., 1997). Deux orientations pouvaient être envisagées: d'une part, l'orientation classique de satisfaction du client pour laquelle il s'agit d'assurer via des procédures la qualité de la réponse aux besoins du client, d'autre part, la structuration du retour d'expérience en vue d'une capitalisation locale et globale des apprentissages. Ces deux orientations impliquent évidemment des contenus différents pour le système d'assurance qualité. Par exemple, il serait tout à fait logique dans une perspective d'apprentissage de demander aux responsables internes de consigner les raisons pour lesquelles ils renoncent dans certains cas à aller au bout du processus de réponse à un appel d'offre (difficultés à maîtriser le sujet, anticipation d'un manque de compétitivité économique, manque de temps, etc.), alors que ces non réponses sortent clairement du champ d'application d'une perspective d'assurance qualité classique. Aussi, si cette entreprise choisit, dans le cadre de sa démarche d'assurance qualité, de restructurer son processus d'élaboration des offres de façon à pouvoir reconstituer toutes les raisons de non aboutissement de ces offres, elle aura privilégié l'apprentissage dans la rationalisation de ce processus opératoire.

On imagine sur ce dernier exemple que les modalités concrètes d'une rationalisation de l'action prenant explicitement en compte 
un objectif d'apprentissage devront nécessairement réaliser un compromis entre deux finalités, celle d'une performance opérationnelle à court terme (comprenant ici la satisfaction de chaque client) et celle d'un développement de nouveaux savoirs (ici sur les raisons d'aboutissements du processus d'élaboration des offres) susceptibles d'engendrer des résultats à plus long terme. En effet, vouloir concevoir le système pour qu'il répondre parfaitement aux deux objectifs déboucherait sur un système trop lourd à faire fonctionner, d'où la nécessité d'un compromis. L'important est déjà qu'une telle question soit posée, et qu'on s'efforce de hiérarchiser les différentes finalités et de les prendre en compte simultanément sous la forme d'un compromis, expression locale de la stratégie de l'entreprise. À partir du moment où dans cette stratégie on fait une bonne place à l'objectif d'apprentissage, on ouvre la voie à une démarche de rationalisation simultanée de l'action et de l'apprentissage.

Au-delà de la spécificité de la présence explicite de cet objectif de rationalisation des apprentissages au sein d'une démarche de rationalisation de l'action, nous allons maintenant caractériser davantage les formes possibles d'une telle rationalisation simultanée.

\section{III. - LA RATIONALISATION SIMULTANÉE \\ DES MODALITÉS DE NATURE INSTRUMENTALE OU DE STRUCTURATION ORGANISATIONNELLE}

Il s'agira ici de mobiliser la dichotomie classique entre les démarches instrumen- tales et celles portant sur la structuration organisationnelle. Les premières sont directement concernées par la formalisation des savoirs de l'action; alors que les secondes portent plus spécifiquement sur la structuration des rôles et des relations entre les acteurs en situation d'apprentissage.

\section{Les « outils d'apprentissage »}

Par outils d'apprentissage, nous entendons les outils de gestion dont la mise en œuvre vise aussi explicitement à aider leurs utilisateurs à mieux maîtriser et à développer les savoirs-clés de l'action, notamment en pointant ceux qui posent problème et en structurant le cadre d'analyse. Ces outils peuvent servir en particulier à la prise de décision ou à la mémorisation de cette dernière, et intègre donc des fonctions de capitalisation.

Nous avons proposé le concept d'outil d'apprentissage pour caractériser les spécificités d'un outil de gestion du risque bancaire que nous avions élaboré en réponse à une demande d'implantation d'un outil d'aide à la décision concernant les entrées en relation avec des «professionnels » (artisan, petite entreprise, commerçant). Il s'était avéré que ce problème était un des plus complexes en matière de gestion du risque bancaire du fait qu'il mélangeait intimement les risques liés à la création ou reprise d'entreprise (expérience, compétence, rentabilité, etc.) et ceux liés aux comportements des particuliers (personnalité, environnement familial, patrimoine, etc.). Le taux de mortalité élevé de ces entreprises en phase de création (50\% durant les 5 premières années de vie) prouve la fragilité de ces affaires et la difficulté d'apprécier les risques. Ces derniers 
ne peuvent être évalués en utilisant les méthodes statistiques classiques, basées sur des données objectives. En effet, contrairement à d'autres types de dossiers, les critères de mesure des risques sont des critères de jugement sur le client (sérieux, compétences, etc.) et sur son projet (réalisme de la rentabilité prévisionnelle, sensibilité du chiffre d'affaires, etc.) Aussi, nous avons proposé un outil qui modélisait l'expertise nécessaire à l'appréciation du risque. Celui ci se présentait sous la forme d'une grille arborescente d'évaluation et d'agrégation des différents critères de risque, débouchant sur une évaluation globale de chaque dossier. Ses modalités d'usage, en particulier structuration des entretiens avec le client et support au processus de décision collégial, traduisaient sa véritable vocation: il offrait avant tout une aide au raisonnement d'évaluation, qui permettait de rendre explicite dans le processus de décision, les raisons de l'acceptation ou du refus d'un dossier, et en cela de favoriser les apprentissages individuels et collectifs. Il s'agissait donc bien simultanément d'améliorer à court terme le processus de décision, d'offrir un support au développement d'une doctrine collective en matière de gestion du risque sur ce segment d'activité; et d'instituer une boucle d'apprentissage par la mise en relation des raisons mémorisées d'une acceptation avec le destin ultérieur du dossier.

Pour plus de détails sur cette expérience et sa conceptualisation, nous renvoyons le lecteur à Sardas et Touati (1997). Nous verrons également plus loin une autre application de cette démarche dans le domaine industriel.

Bien entendu, ces outils d'apprentissage doivent être pensés et conçus dans cet esprit. À ce niveau, signalons que les outils d'apprentissage ne s'apparentent pas aux systèmes experts. Même si ces derniers se veulent être un moyen de transfert et de capitalisation de l'expertise, il se trouve dans les faits que ces systèmes experts présentent souvent de graves lacunes sur le plan pédagogique, au sens où leur maniement ne permet pas d'en comprendre la logique et donc favorise peu les apprentissages. Une explication plausible serait de dire que les concepteurs de systèmes experts ont trop souvent envisagé les modules d'explication des raisonnements comme un simple dépouillement de la trace des règles activées. Or, ces traces ne répondent pas aux besoins cognitifs de l'utilisateur car ces explications s'attachent essentiellement à retourner les règles utilisées dans le cas particulier du problème posé au lieu d'expliciter les principes généraux de fond ayant présidé à l'élaboration de ces règles.

De la même manière, notre concept d'outils d'apprentissage ne se confond pas avec celui introduit par des auteurs tels que Fulmer et al. (1998). Ces derniers ont proposé une classification de ces outils; en distinguant par exemple, les utilitaires (exemple: sondages) des outils de prévision (exemple: analyse de scénarios). En effet, à la différence de ces auteurs qui assimilent les outils d'apprentissage aux outils tout court, la notion d'outils d'apprentissage que nous proposons renvoie à une autre génération d'outils, orientée structurellement vers l'apprentissage.

Pour préciser davantage ce concept d'outil d'apprentissage, nous allons le mettre en perspective par rapport à d'autres notions qui lui sont proches, gravitant toutes autour du thème de l'apprentissage. En particulier, 
nous confronterons les outils d'apprentissage aux outils coopératifs, aux outils d'aide au raisonnement qualitatif et aux concepts pragmatiques.

\section{Outils d'apprentissage versus outils coopératifs}

Défini simplement, un outil coopératif (Sabroux et Zaraté, 1995) fait appel à quatre principes, à savoir: l'interactivité, la symétrie entre l'utilisateur et le système en question et enfin, la prise en compte des caractéristiques de l'utilisateur final (entre autres ses compétences) et du contexte organisationnel (exemple: niveau hiérarchique de l'utilisateur) associé au processus de résolution du problème.

En prenant connaissance de certains travaux de ce courant de recherche ${ }^{5}$, il nous semble que ce concept est le messager d'une philosophie particulière: la notion de coopération est en quelque sorte assimilée à une participation du système au processus de prise de décision. Le système intervient lorsque l'utilisateur commet une erreur, et vice versa.

À titre d'exemple, l'outil sert à avertir l'utilisateur d'une incompréhension de la décision prise en se basant sur les modèles de connaissance qu'il détient. Aussi, on peut penser que le but premier de ces instruments est davantage de compléter le champ d'action de l'utilisateur que de l'encadrer, ce qui distingue donc les outils coopératifs des outils d'apprentissage. Leur usage simultané n'est toutefois pas exclu; bien au contraire puisque l'on aura tout intérêt, lorsque cela est possible, à intégrer les outils coopératifs dans le cadre global proposé par les outils d'apprentissage.

\section{Outils d'apprentissage versus outils d'aide au raisonnement qualitatif}

Les systèmes d'aide au raisonnement qualitatif (Forgionne et Clements, 1995) ont été conçus en vue d'apporter une assistance au décideur à chacune des phases du processus de décision, en l'occurrence, les phases d'intelligence, de design et de choix (Simon, 1983). Se présentant sous la forme d'une boite à outils regroupant plusieurs fonctionnalités (organiser les idées, simuler des altérations des solutions proposées, etc.), ces systèmes devraient offrir en théorie une aide appréciable en termes d'apprentissage. Malheureusement les auteurs ne fournissent aucun exemple concret d'une telle démarche.

\section{Outils d'apprentissage versus concepts pragmatiques}

Les concepts pragmatiques ont été introduits par les chercheurs et praticiens issus du domaine de la formation professionnelle (voir notamment Samurçay et Pastré (1995) pour structurer et formaliser les savoirs de référence correspondant à une compétence donnée. Les concepts pragmatiques jouent un double rôle: d'une part, ils organisent l'action efficace en permettant un couplage fort entre les prises d'information effectuées par le sujet et les opérations qu'il exécute; d'autre part, ils permettent également à sa représentation fonctionnelle de se structurer en mettant l'accent sur les relations essentielles existant entre les variables de la situation. Ces auteurs ont eu

5. Cf. Sabroux et Zaraté (1995), Smyth (1995), Brezillon et Cases (1995). 
recours à cette logique d'instrumentation pour rationaliser la conduite de processus de production continus, non complètement automatisés et caractérisés par de longs délais de réponse, très difficilement contrôlables par l'opérateur. Tels qu'ils sont présentés, ces concepts pragmatiques cherchent à développer les compétences d'analyse des opérateurs en les aidant à structurer leur raisonnement. En cela, on peut affirmer qu'ils sont de véritables outils d'apprentissage.

Arrivant au terme de notre tentative de clarification du concept d'outil d'apprentissage, il nous reste à répondre à la question suivante: en quoi ces outils recouvrent d'un point de vue historique aujourd'hui, un nouveau mode de rationalisation, tel que défini par Hatchuel et Weil (1992)? Ces auteurs analysent les techniques managériales (recherche opérationnelle, systèmes experts, gestion de production assistée par ordinateur, etc.) comme des vagues de rationalisation. À titre d'exemple, l'apparition des systèmes experts correspondrait à une vague de rationalisation caractérisée par une philosophie gestionnaire (l'automatisation de l'expertise), par un substrat technique (base de connaissances activée par un moteur) et enfin, par une figure d'acteur (le cogniticien).

Concernant spécifiquement les outils d'apprentissage, nous pensons pouvoir affirmer que ceux-ci renvoient à une nouvelle vague de rationalisation orientée structurellement vers l'apprentissage. Ces démarches de rationalisation ont comme point commun d'une part, de privilégier une métamodélisation des processus de décision, et d'autre part, d'être accompagnées par un acteur méthode, jouant le rôle d'animateur méthodologue. Ce rôle d'animation comporterait notamment des responsabilités de formation, de repérage des difficultés éprouvées par les sujets apprenants, de capitalisation et de diffusion des apprentissages locaux. Aussi, cette logique d'instrumentation des processus d'apprentissage se traduirait par l'émergence d'une nouvelle figure d'acteur, différente de celle de l'acteur méthode prescripteur traditionnel.

L'exemple ci dessous extrait des travaux d'Aggeri et al. (1995b) illustre lui aussi cette forme d'instrumentation des apprentissages. La présentation de cet exemple est précédée par une description du contexte dans lequel s'insère cette forme de rationalisation, et ce dans le but de bien saisir les liens entre la nature de l'activité et la nature de l'instrument mis en place.

Un exemple d'outil d'apprentissage: le recours à un outil d'aide au raisonnement pour l'évaluation de filières de recyclage

L'intégration de la contrainte de recyclage dans l'industrie automobile n'est guère évidente. D'une part, l'utilisation de matériaux recyclables peut rentrer en conflit avec d'autres aspects de la qualité (exemple, problèmes d'aspect et de contraintes mécaniques fortes) ou d'autres valeurs environnementales. Ainsi, le recours aux matières plastiques détériore la re-cyclabilité mais réduit le poids du véhicule et donc la consommation en combustibles. D'autre part, il existe de nombreuses limites techniques au recyclage dues entre autres à la variété et complexité des matériaux dans l'automobile, aux difficultés de démontage et de séparation des pièces. De même, la viabilité économique du recyclage est précaire car en dehors des métaux, les débouchés rentables et durables se limitent à quelques matériaux. 
Sans idée précise d'un schéma de traitement des véhicules hors d'usage viable techniquement et économiquement, il était difficile d'imposer des mesures de normalisation, et de définir des quotas de recyclage sans entraîner des déséquilibres importants dans la situation économique des filières de recyclage, et par voie de conséquence dans la compétition entre matériaux. Aussi, au lieu d'introduire une réglementation coercitive non fondée sur des savoirs probants, un accord cadre a été signé entre différents acteurs concernés en vue d'encourager la recherche et développement et de renforcer les coopérations entre constructeurs automobiles et autres partenaires du réseau (les démolisseurs, les broyeurs, les fournisseurs, etc.). Cet accord cadre constituait également pour l'État un moyen privilégié de mieux comprendre les contraintes du recyclage.

Pour mettre en œuvre cet accord cadre, une grande entreprise française a dû s'attaquer à deux objectifs (Aggeri et al., 1995b) :

- un pôle conception qui s'attache à introduire la valeur du recyclage dans les projets de conception de véhicules;

- un pôle valorisation qui évalue systématiquement les filières de valorisation sachant que la réussite relative de certaines filières favorise des orientations fortes en conception.

C'est ce dernier pôle qui nous intéresse ici en raison de la forme de rationalisation dont il a fait l'objet. Cette forme de rationalisation a été proposée par des chercheurs du Centre de gestion scientifiques, jouant un rôle d'accompagnateurs dans ce processus de changement. La démarche retenue pour évaluer les filières (une filière étant définie comme un couple matériau pièce), a consisté à reprendre les principes de l'outil d'aide au raisonnement (présenté plus haut). Ce dernier permet d'évaluer la filière en attribuant une notation qualitative (insuffisant, moyen inférieur, moyen supérieur, bon) à des critères de jugement prédéterminés. La nécessité de recourir à une analyse qualitative de type « jugement » est encore une fois cohérente avec l'incertitude qui entoure l'activité. Cette grille méthodologique est fondée sur une structuration hiérarchique des critères d'analyse construite autour de six grandes rubriques:

- les types de filières: retour à l'automobile, réemploi hors de l'automobile, valorisation énergétique,

- les stades des filières: R\&D, amorce, développement, efficience,

- la robustesse du réseau industriel: cette notion recouvre plusieurs critères qui permettent de cerner l'engagement des acteurs, la connaissance disponible sur les procédés et les transactions, l'ouverture internationale, - la rentabilité des étapes de valorisation: cette notion porte sur les gisements prévisibles, les débouchés, les rentes par acteur (extraction, transformation, broyeurs, etc.), - les possibilités de contrôle: on examine les possibilités de contrôle de l'entreprise en question sur les acteurs et les transactions de la filière,

- l'impact réglementaire: on s'intéresse ici aux évolutions possibles et connues des réglementations touchant particulièrement la filière.

On constate ainsi que cet outil privilégie une structuration du champ d'analyse en un ensemble restreint de critères; ce qui permet par la suite de maîtriser la complexité des raisonnements. En parallèle à cette délimitation, l'outil propose des règles d'agrégation des évaluations partielles sur chacun des critères. Ces règles d'agrégation se veu- 
lent être avant tout transparentes et intelligibles. À titre d'illustration, l'outil préconise que si l'évaluateur juge que la filière examinée est insuffisante sur un des critères, cela l'oblige à considérer la filière comme insuffisamment recyclable dans son ensemble.

La mise en œuvre de cet outil d'évaluation des filières de recyclage est supportée par une nouvelle figure d'acteur, en l'occurrence l'expert du recyclage. Le rôle de ces experts a été institutionnalisé en créant un département de recyclage.

\section{L'intervention sur les structures organisationnelles}

Cette forme de rationalisation cherche à structurer les rôles des acteurs et leurs relations à travers un redécoupage organisationnel de l'activité de façon à renforcer les apprentissages croisés de ces derniers. L'enjeu ici n'est pas d'améliorer la réactivité ou l'efficacité des fonctionnements par un équilibrage approprié des exigences de différenciation et d'intégration de l'organisation (Lawrence et Lorsch, 1989) mais d'optimiser les apprentissages. Bien entendu certains de ces objectifs ne sont pas forcément exclusifs. Cependant en mettant l'accent sur l'apprentissage plutôt que sur la réactivité, ceci signifie en particulier que les choix effectués dans les actions quotidiennes ne doivent pas se faire au détriment de l'investigation et qu'ils doivent s'inscrire dans une politique technique claire; ce qui n'est pas le cas par exemple pour les structures projet, structures visant par excellence la réactivité, qui sont plus orientées vers une logique de compromis local.
Ainsi, si on reprend la vision théorique de Galbraith (1995) qui identifie cinq formes d'organisation (fonctionnelle, par produit, par marché, géographique ou par process) dont chacune est contingente à certaines caractéristiques de l'activité (la variété des produits, le délai de réponse au marché, la vitesse du changement, etc.), on peut penser que les restructurations organisationnelles orientées vers l'apprentissage ne se retrouvent pas forcément dans cette typologie dans la mesure où les déficits de savoirs auxquels les redécoupages dont il est question veulent répondre peuvent concerner, non pas une fonction, ni un process dans sa globalité mais certains problèmes spécifiques.

Pour donner une idée plus claire de cette modalité d'intervention au niveau structurel, nous nous référerons à une expérience fort intéressante en la matière, présentée dans (Lefebvre et Sardas, 1995a).

Un exemple d'organisation orientée vers l'apprentissage: la mise en place du CCC dans une entreprise aéronautique

Cette entreprise aéronautique a commencé à expérimenter depuis plusieurs années l'usage des matériaux composites dans ces produits. Contrairement aux matériaux métalliques auxquels ils viennent se substituer, les matériaux composites permettent une créativité pratiquement infinie des concepteurs, qui peuvent imaginer des formes très complexes avec des épaisseurs variables. L'enjeu est notamment d'obtenir des gains de poids avec des propriétés mécaniques comparables voire supérieures à celles des matériaux métalliques, et de pouvoir innover du point de vue de l'architecture du produit en remplaçant plusieurs 
pièces métalliques par une seule pièce en composites. Le procédé de fabrication est à la fois simple, il s'agit en particulier de superposer diverses couches de matières premières et de les presser à chaud pour obtenir une pièce moulée en composites, mais en fait très difficile à maîtriser et à reproduire de façon stable. La mise au point de la conception d'une pièce se fait alors nécessairement en même temps que la mise au point de son process de fabrication, la conception du produit et du process étant beaucoup plus interdépendantes que pour les matériaux métalliques.

L'usage des matériaux composites apparaissait donc très prometteur mais encore bien loin d'être maîtrisé. C'est pourquoi la décision de développer l'usage des composites a été accompagnée d'une innovation organisationnelle destinée à accélérer les apprentissages afin d'obtenir plus rapidement les gains attendus tout en maîtrisant mieux les risques associés à cette innovation technique.

Comme son nom l'indique, le caractère innovant de cette structure organisationnelle, baptisée Centre de compétences en composites, réside dans sa finalité, à savoir l'accélération des apprentissages dans l'utilisation industrielle des matériaux composites. Pour autant, il ne s'agit pas d'une entité exclusivement dédiée à l'élaboration de nouveaux savoirs. Cette entité a en charge l'ensemble de l'activité relative au développement de pièces en composites. Dans cette double optique, il a été décidé de rassembler en un même lieu les quatre fonctions essentielles au développement des pièces et au développement de la maîtrise de l'emploi des matériaux composites: le laboratoire (qui étudie et teste les nouveaux matériaux), le bureau d'études, le bureau des méthodes (qui assure également la fabrication de certaines pièces de développement), et la qualité (sachant que la fabrication série de ces pièces est entièrement sous-traitée). Notons que cette entité dispose de moyens propres d'expérimentation. Les effectifs affectés à cette structure, sélectionnés en fonction de leur expertise technique, de leur créativité, et de leur capacité à œuvrer dans un groupe interdisciplinaire, travaillent sur plusieurs projets de développement, sur des projets exploratoires et ont des activités de recherche et de capitalisation métier. Ce dernier point est particulièrement important. Il faut en effet savoir que si on avait toujours exhorté les ingénieurs à consigner - à l'occasion de mesures de laboratoires, de conception d'une pièce ou de mise au point d'un procédé - les choix de méthodes effectués, les paramètres retenus, les raisons de ces choix, comme les résultats obtenus, bien peu le faisait de manière systématique et encore moins de manière détaillée, si bien que l'accumulation de savoirs était plus individuelle qu'organisationnelle. La mise au point de la nouvelle organisation fût l'occasion d'une certaine rationalisation, partielle encore, de ces procédures de capitalisation. Outre l'établissement de jalons de projet, l'engagement des acteurs à ces divers jalons reposait sur une base écrite dans laquelle les problèmes à résoudre, les missions de chacun et les choix de méthode étaient consignés; la résolution de ces problèmes et les succès ou errements de telle ou telle orientation devaient être suivis par écrit et une réorientation devait à nouveau faire l'objet d'un engagement écrit des acteurs.

Par ailleurs, pour permettre une diffusion des savoirs développés au sein des métiers, on inventa de nouveaux dispositifs 
d'échange de connaissances. À titre d'illustration, pour contrecarrer l'effet centrifuge $\mathrm{du}$ fonctionnement en projets, on institua des réunions de présentation au sein du bureau d'études, suivies de discussions et faisant l'objet d'une formalisation écrite des petites trouvailles de conception.

Enfin, pour animer globalement ces nouveaux modes de fonctionnement, un coordonnateur a été nommé parmi les responsables des quatre groupes: son rôle est d'organiser la coordination et le suivi des différents projets, d'allouer les moyens techniques communs aux quatre groupes et enfin de représenter le CCC au sein des différentes instances de direction de l'entreprise. Soulignons que ce coordonateur n'a pas de prérogative hiérarchique sur les équipes en présence. En effet, tout en étant une entité reconnue dans l'organigramme de l'entreprise, le CCC ne vient pas se substituer aux directions d'origine des services qui composent ce dernier. C'est pourquoi, nous pensons que cette étude de cas est particulièrement intéressante dans la mesure où le dispositif mis en place est hybride, se situant entre le structurel et le dispositif de coordination.

Soulignons pour finir que, sans qu'il y ait de référence explicite à une démarche de knowledge management, qui à l'époque était encore peu répandue, plusieurs des procédures mises en place au sein de cette nouvelle organisation sont bien du registre de la gestion des connaissances. Cependant, contrairement aux démarches génériques actuelles de KM très orientées vers la for- malisation des savoirs à des fins de réutilisation, il s'agit ici davantage de créer de nouveaux savoirs. De plus alors que les démarches de KM s'appuient fortement sur la mise en place de systèmes d'information dédiés à la capitalisation, nous avons dans cet exemple, tant pour l'organisation que pour les procédures, une forte imbrication entre les dispositifs de gestion de l'activité et ceux de gestion des connaissances ${ }^{6}$. Ainsi par exemple, les fiches opératoires élaborées par les préparateurs méthodes pour la fabrication interne (des préséries de pièces en développement qui permettent la mise au point) ont été conçues pour être à la fois des guides opératoires et des supports de saisie des réglages réels et des différents événements rencontrés en cours de fabrication. Ces documents viennent ensuite nourrir des réunions transverses où les expériences sont échangées (entre concepteurs du produit, concepteurs du process, responsables qualité et ingénieurs du laboratoire) et des solutions aux problèmes rencontrés sont recherchées collectivement. On voit ici comment rationalisation de l'activité et rationalisation de l'apprentissage s'interpénètrent fortement dans cette nouvelle organisation.

\section{CONCLUSION}

On pourrait donner d'autres exemples de rationalisation simultanée de l'action et des apprentissages. Cela étant, les exemples présentés dans cet article prouvent à nos yeux que les entreprises, dont les activités

6. Concernant la nécessaire intégration des démarches de KM dans des approches de gestion plus globales des activités, nos travaux sur la transformation des métiers de conception (cf. Lefebvre et al., 2003) mettent en évidence la nécessité d'éviter d'isoler la gestion des connaissances (tant les systèmes de KM que les rôles d'experts) de l'activité courante de conception. 
sont relativement complexes, sont en train de vivre un tournant dans leurs modes de rationalisation de l'action dans le sens où ces derniers sont de plus en plus pensés en fonction de leurs potentialités d'apprentissage. Ceci laisse entendre que les transformations engagées, qu'elles concernent de nouveaux outils ou de nouvelles organisations, sont elles-mêmes plus complexes à concevoir et à mettre en œuvre.

Il faut en particulier suivre ces expériences et analyser finement la réalité des évolutions. Même s'ils ont été conçus de façon participative, les outils ou dispositifs organisationnels mis en œuvre ne constituent en effet au mieux que de nouveaux cadres censés être pertinents pour l'action et les apprentissages. Il revient ensuite aux acteurs opérationnels de leur donner vie, de les adapter à l'activité réelle et de les faire évoluer. Seule une analyse fine des nouveaux fonctionnements permet de savoir si ces cadres jouent bien leur rôle et qu'on aboutit bien à une amélioration des performances et des apprentissages. Bien entendu, en fonction des enseignements, on peut être amené à réorienter et à remettre à jour autant que nécessaire ces formes de rationalisation. De par notre expérience de chercheurs intervenants ayant été engagés dans de telles démarches, nous insistons fortement sur l'importance de cette phase d'accompagnement de la mise en œuvre, car cet enjeu est souvent minimisé par les dirigeants responsables des changements. Cela exige de la part des intervenants internes et externes de réelles capacités à interpréter les fonctionnements organisationnels.

Ceci nous ramène en particulier au rôle-clé joué par le nouvel acteur méthodologue dont nous avons mis en évidence plus haut l'émergence. Il reste encore du chemin à faire avant que le statut et les missions d'un tel acteur ne se précisent, et qu'il dispose d'une reconnaissance suffisante. C'est pourtant une condition indispensable pour que des démarches de rationalisation simultanée de l'action et des apprentissages aient une portée réelle.

\section{BibliographIE}

Aggeri F., Hatchuel A., Lefebvre P., « La naissance de la voiture recyclable: intervention de l'État et apprentissages collectifs », Cahier de recherche, centre de gestion scientifique, mars 1995a.

Aggeri F., Hatchuel A., Lefebvre P., « Innovation produit et fin de vie », Rapport de travail, École des mines de Paris, novembre 1995b.

Amadieu J. F., Cadin L., Compétence et organisation qualifiante, Economica, Paris, 1996. Argyris C. H., Schon D., Organizational learning: a theory of action perspective, Reading Addison-Wesley, 1978.

Bonnis J., «Les organisations formantes: pourquoi et à quelles conditions », Personnel, n 329, 1992, p. 6-10.

Brézillon P., Cases E., "Cooperating for assisting intellingently operators”, Actes du colloque, Conception des systèmes coopératifs, Antibes, janvier 1995. 
CERI (Centre pour la recherche et l'innovation dans l'enseignement), Société du savoir et gestion des connaissances, Paris, OCDE, 2000.

Daft R., Weick K. E. “Toward a Model of Organizations as Interpretation Systems”, Academy of management review, vol. 9, $\mathrm{n}^{\circ}$ 2, 1984, p. 284-295.

David A., Lefebvre Ph, Sardas J.C., «Enjeux et impact de la mise sous assurance qualité d'une activité complexe et en forte croissance », École des mines, 1997.

Dierkes M., Berthoin Antal A., Child J., Nonaka I., Handbook of organizational learning \& knowledge, Oxford, Oxford University Press 2001.

Erschler J., Thuriot C., « Approche par contraintes pour l'aide aux décisions d'ordonnancement », Les nouvelles rationalisations de la production, 1992.

Fiol C. M., Lyles M. A., "Organizational Learning”, Academy of management review, vol. $10, \mathrm{n}^{\circ} 4,1985$, p. 803-813.

Forgionne G. A, Clements J. P., "Qualitative thinking support systemps”, Revue des systèmes de décision, vol. 4, $\mathrm{n}^{\circ}$ 2, 1995, p. 103-137.

Freund J., Études sur Max Weber, Genève, Droz, 1990.

Fulmer R. M., Gibbs P., Keys J. B., “The second generation learning organizations: new tools for sustaining competitive advantage", Organizational Dynamics, automne, 1998, p. 7-19.

Galbraith Jay R., Designing organizations. An executive briefing on strategy, structure, and process, Jossey Bass Publishers, San Fransisco, 1995.

Hatchuel A., «Apprentissages collectifs et activités de conception », Revue française de gestion, Juin-Août 1994, p. 109-120.

Hatchuel A., «L'avenir de la grande entreprise industrielle: vers une rationalisation des processus d'apprentissage ? », Entreprises et Histoires, n 10, 1995.

Hatchuel A., Moisdon J-C., « Modèles et apprentissage organisationnel », Cahiers d'économie et sociologie rurales, $\mathrm{n}^{\circ} 28,1993$, p. 18-32.

Hatchuel A., Weil B, L'expert et le système: gestion des savoirs et métamorphose des acteurs dans l'entreprise industrielle suivi de quatre histoires de systèmes-experts, Paris, Économica, 1992.

Hedberg B.L.T., "How organizations learn and unlearn", Handbook of organizational design, Oxford University Press, New York, 1981, p. 3-27.

Huber G. P., "Organizational learning: the contributing processes and the literatures", Organizational learning, Sage Publications, London, 1996, p. 124-162.

Jeantet A., Tichkievitch S., Mer S., « Les objets intermédiaires de la conception: modélisation et communication », Le Communicationnel pour concevoir, Europa Productions, Paris. 1995.

Lawrence P., Lorsch J., Adapter les structures de l'organisation, Les Éditions d'Organisation, Paris, 1989.

Lefebvre P., Sardas J-C., « Transformations organisationnellles et recomposition des modes d'apprentissage », Actes, Colloque La connaissance dans la dynamique des organisations productives, Aix-Marseille, 14-15 septembre 1995. 
Lefebvre P., Roos P., Sardas J. C, « Gestion des compétences, gestion des connaissances et enjeux identitaires en conception: pour une approche unifiée de la dynamique métier », Compétences et connaissances dans les organisations, Guénette A. M., Rossi M. et Sardas J. C. (dir.), Édition SEES, Lausanne, janvier 2003.

Lefebvre L.-A, Lefebvre E., Mohnen P., La conduite des affaires dans l'économie du savoir, Paris, Economica, 2001.

Levitt B., March, J. G., “Organizational Learning”, Annual review of sociology, n 14, 1988, p. 319-340.

March J.G, "Exploration and Exploitation in Organizational Learning", Organization science, vol. 2, $\mathrm{n}^{\circ} 1,1991$, p. 71-87.

Midler C., L'auto qui n'existait pas. Management des projets et transformation de l'entreprise, Interédition, Paris 1993.

Moisdon J.C., Weil B., «L'invention d'une voiture: un exercice de relations sociales », Gérer et Comprendre, Annales des Mines, septembre-décembre 1992.

Mussano J.P., «Organisation et développement des compétences: vers des organisations qualifiantes », Entreprise et personnel, avril 1992.

Nakhla M., Soler L.G., « Pilotage de projet et contrats internes », Revue française de gestion, septembre-octobre 1996.

Ponssard J.P., Tanguy H., "Planning in firms as an interactive process", Theory and Decision, 34, 1993, p. 139-159.

Peyrard C., «Technologies de conduite et technologies intellectuelles: la production de connaissances dans les activités de travail », Éducation permanente, $\mathrm{n}^{\circ} 112$, 1992, p. 31-37.

Pine B.J., Mass customization: the new frontier in business competition, Harvard Business School Press, Boston, MA, 1993,

Sabroux C.R., Zarate P., "Coopération typology for decision support", Actes, colloque Conception des systèmes coopératifs, janvier 1995, Antibes.

Samurçy R., Pastré, P., « La conceptualisation des situations de travail dans la formation des compétences », Education Permanente, n 123, 1995.

Sardas J. C, Touati N., « Processus d'apprentissage et gestion du risque bancaire », Systèmes d'information et management, décembre, 1997, p. 87-121.

Shrivastava P., Schneider S., "Organizational Frames of Reference”, Human Relations, vol. 37, $\mathrm{n}^{\circ}$ 10, 1984, p. 795-809.

Simon H., Administration et processus de décision, Economica, Paris, 1983.

Smyth M., "Human computer co-operative systems empowering users througn parttnership”, Actes, colloque Conception des outils coopératifs, Antibes, 1995.

Veltz P., Zarifian Ph., « De la productivité des ressources à la productivité par l'organisation », Revue française de gestion, janvier-février 1994, p. 59-66.

Zarifian Ph., Le modèle de la compétence, Éditions Liaisons 2001. 